\title{
Response of cerebral blood flow and cerebrovascular reactivity to acetazolamide in patients with dementia and idiopathic normal-pressure hydrocephalus
}

\section{Chia-Cheng Chang, M.D., Nobumasa Kuwana, M.D., Susumu Ito, M.D., and Tadashi Ikegami, M.D.}

Departments of Neurosurgery and Radiology, Yokohama Minami Kyosai Hospital, Yokohama, Japan

The responses of cerebral blood flow $(\mathrm{CBF})$ and cerebrovascular reactivity $(\mathrm{CVR})$ to administration of acetazolamide were investigated in 16 patients with dementia and ventriculomegaly to clarify the cerebral hemodynamics in patients with idiopathic normal-pressure hydrocephalus (NPH).

The mean $\mathrm{CBF}$ velocity in the whole brain was measured by the Patlak plot method by using technetium-99m hexamethylpropyleneamine oxime single-photon emission computerized tomography. The CVR values were obtained from the response to administration of $500 \mathrm{mg}$ of acetazolamide and calculated as the percentage of change from the baseline mean $\mathrm{CBF}$ value. The mean $\mathrm{CBF}$ value was significantly reduced $(\mathrm{p}<0.01)$ in six patients with $(35.2 \pm 5 \mathrm{ml} / 100 \mathrm{~g} / \mathrm{minute})$ and 10 patients without $(33.5 \pm 2.8 \mathrm{ml} / 100 \mathrm{~g} / \mathrm{minute}) \mathrm{NPH}$ compared with the age-matched normal controls $(40.8 \pm 3.2 \mathrm{ml} / 100$ $\mathrm{g} / \mathrm{minute}$ ), showing no significant difference. The CVR was significantly impaired in patients with NPH $(0.8 \pm 1.7 \% ; \mathrm{p}<0.001)$, whereas in patients without NPH preserved CVR $(11.3 \pm 3 \%)$ was demonstrated compared with the normal controls $(14.7 \pm 1.1 \%)$. In patients with NPH a significantly lower CVR (p < 0.001) was shown than in those without NPH. The CVR significantly increased $(\mathrm{p}<0.001)$ after placement of a shunt in patients with NPH.

Reductions in both CBF and CVR may be diagnostic indicators of NPH in patients with dementia in whom ventriculomegaly is present. In patients with dementia and idiopathic NPH both reduced CBF and extremely impaired CVR are shown. The results of the present study suggest that ischemia due to the process of NPH is responsible for the reduction of CBF and manifestation of symptoms in patients with idiopathic NPH.

Key Words * cerebral blood flow * cerebrovascular reactivity * acetazolamide * dementia * idiopathic normal-pressure hydrocephalus

Idiopathic normal-pressure hydrocephalus (NPH) is an important cause of dementia in elderly patients. The identification of NPH as a major contributing cause of dementia is difficult in patients with dementia of mixed causes, such as idiopathic NPH associated with multiple-infarct dementia or senile dementia of 
the Alzheimer's disease type. Ventriculomegaly may be related to the atrophic process or to true hydrocephalus with a cerebrospinal fluid (CSF) absorption deficit. The resultant ventricular dilation is difficult to relate to a true hydrocephalic process, and to what extent hydrocephalus contributes to the neurological deficits is uncertain.

In patients with NPH reduced cerebral blood flow (CBF) is generally shown, [6,7,9] but the observation of reduced $\mathrm{CBF}$ is not sufficient to identify NPH in patients with dementia because such a reduction is also observed when cerebral metabolism is decreased.

Acetazolamide is known to increase CBF without changing the cerebral metabolic rate of oxygen.[14,17] Patients with compromised circulation and resultant reduced $\mathrm{CBF}$ due to reduced perfusion pressure do not respond to acetazolamide.[19] However, little is known about the cerebrovascular reactivity (CVR) in patients with idiopathic NPH. In an experimental study of animals with hydrocephalus it was shown that impairment of the cerebral microcirculation results from the collapse of and ultimately reduced numbers of capillaries in the periventricular tissue.[4] Mechanical stretching of the anterior cerebral arteries and veins over the corpus callosum and compression of the cerebral microcirculation by the enlarged ventricles in NPH would limit the capacity of these vessels to constrict or dilate.[12] Increased water content and high tissue pressure in the periventricular white matter due to increased bulk diffusion of CSF through the brain interstitium would also impair the CVR in patients with NPH.[15]

In this study we investigated the responses of $\mathrm{CBF}$ and CVR to acetazolamide in patients with dementia and ventriculomegaly to clarify the cerebral hemodynamics in patients in whom dementia and idiopathic NPH are present.

\section{CLINICAL MATERIAL AND METHODS}

\section{Patient Population}

This study included 16 patients with dementia. There were nine men and seven women (age range 42-82 years, mean 70.7 years) with ventriculomegaly of unknown cause. In all patients cognitive and memory disturbances were demonstrated, and all required some supervision at home. Computerized tomography (CT) scanning revealed enlargement of the ventricular system with or without cortical atrophy in all patients. Computerized tomography cisternography was performed in 13 patients after intrathecal injection of iohexol. Evidence of ventricular stasis for more than 24 hours and/or persistent cerebral blush for more than 48 hours was considered abnormal.[5] Magnetic resonance (MR) imaging demonstrated various degrees of white matter lesions in all patients, but no stenosis or obstruction of the main cerebral arteries was detected on MR angiography . Patients with ventricuomegaly of known cause, such as subarachnoid hemorrhage, intraventriclular hemorrhage, trauma, brain tumor, and meningitis, were not included in the present study.

The clinical characteristics of patients before shunt placement are shown in Table 1. Nine patients with progressive symptoms of NPH triad were selected for shunting procedures after a diagnosis of NPH was established. In six of these nine patients a final diagnosis of true idiopathic NPH was made because they responded to the shunt procedure and showed some improvement (Cases 1-6; Table 1). Gait disturbance was improved in five patients, urinary incontinence in four, and dementia in four. The other three patients were considered to not have NPH because they did not respond after shunt placement (Cases 7 , $11,16)$. There were seven patients in whom a shunt placement procedure was not performed and in whom an initial diagnosis of NPH was made: four patients with normal CT cisternography findings and 
no worsening of symptoms (Cases 8, 10,14, and 15); two without worsening of symptoms or increase in ventricular size for 3 years (Cases 9 and 13); and one in whom clinical diagnosis of probable Alzheimer's dementia was made, with normal CT cisternography findings and evidence of decreased CBF in the bilateral temporal and parietal lobes (Case 12). Of these seven patients, the full symptoms of NPH triad were observed in five and dementia only in two. In the end a final diagnosis of NPH was made in six patients (mean age 70.3 years), as compared with 10 patients (mean age 70.9 years) without NPH. There was no difference in the severity of dementia between the patients with and without NPH.

\begin{tabular}{|cccccc|}
\hline \multicolumn{5}{|c|}{ TABLE 1 } \\
CLINICAL CHARACTER STCS OF PATIENTS WITH DEMENT M BEF ORE SHUNT \\
PLACEMENT WITH OR WITHOUT NPH
\end{tabular}

\section{Measurements of $\mathrm{CBF}$ and $\mathrm{CVR}$}

Informed consent was obtained from the patients or their families before the study. The mean CBF and CVR values were measured before the shunt placement procedure. In four patients with NPH further measurements were obtained 2 to 3 months after shunt placement.

The mean CBF values in the whole brain were measured by the Patlak plot method by using technetium-99m hexamethylpropyleneamine oxime (99mTc-HMPAO) single-photon emission CT.[2,10] Following a bolus injection of $740 \mathrm{MBq}$ of 99mTc-HMPAO (Ceretec; Nycomed Amersham, Buckinghamshire, UK) into the right brachial vein, the passage through the heart to the brain was monitored using a rotating scanner (Starcam 400 AC/T, General Electric, Milwaukee, WI). A sequence of 70 frames obtained at 1-second intervals was started in a 128 X 128 format. The hemispherical mean $\mathrm{CBF}$ value was calculated from the hemispherical brain perfusion index by using the linear regression equation of $y=2.75 x+17.7$ between the brain perfusion index and ${ }^{133} \mathrm{Xe}-\mathrm{CBF}$ obtained from the early picture method. The mean $\mathrm{CBF}$ velocity in the whole brain was determined from the mean CBF values 
measured in both hemispheres.

Three days after the baseline study, $500 \mathrm{mg}$ of acetazolamide was injected intravenously, and the mean CBF value was measured 15 minutes later. Measurement of the CVR was obtained from the response to acetazolamide administration, and it was calculated as the percentage of change from the baseline mean CBF value. The conditions of the patients did not change during these 3 days. Our previous preliminary study indicated that the mean $\mathrm{CBF}$ value was not significantly changed over a 3-day period if the condition of the patient remained unchanged.

Ten patients ranging in age from 41 to 83 years (mean age 67.4 years) with no symptoms of NPH and in whom no abnormality was demonstrated on MR imaging were also studied to provide normal values for CVR.

Values are expressed as means \pm standard deviations. Differences between groups were evaluated by one-way analysis of variance with post-hoc analysis in which Scheffé's test was used. Probability values of less than 0.05 were considered significant.

\section{RESULTS}

The mean CBF and CVR values prior to shunt implantation are shown in Tables 1 and 2. The mean CBF value measured in the patients with NPH was not significantly different from that in the patients without $\mathrm{NPH}$. The mean CBF values of both groups were significantly reduced $(\mathrm{p}<0.01)$ compared with the age-matched controls with alert consciousness. The mean CVR value was significantly reduced ( $\mathrm{p}<$ 0.001 ) in the patients with NPH but was not significantly reduced in those without NPH compared with the normal controls. The CVR value in patients with NPH was significantly lower $(p<0.001)$ than in those without NPH. After shunt placement, the mean CVR value in the four patients with NPH significantly increased from $1.4 \pm 1.4 \%$ to $6.5 \pm 1 \%$ ( $\mathrm{p}<0.001$ ). The mean CBF velocity measured in these patients also showed a slight, but not significant, postoperative increase, from $34 \pm 4.6 \mathrm{ml} / 100$ $\mathrm{g} /$ minute to $37.7 \pm 2.5 \mathrm{ml} / 100 \mathrm{~g} /$ minute.

\begin{tabular}{|c|c|c|c|}
\hline Mean CBF and CvR & $\begin{array}{r}\text { Table } 2 \\
\text { des after ad } \\
\text { placement }\end{array}$ & $\begin{array}{l}\text { nistration of a } \\
\text { a shunt }\end{array}$ & tazolamide \\
\hline Measurement & Control & W/O NPH & WI NPH \\
\hline $\begin{array}{l}\text { CBF (mV100 glminute) } \\
\text { CVR }(\%)\end{array}$ & $\begin{array}{l}40.8 \pm 3.2 \\
14.7 \pm 1.1\end{array}$ & $\begin{array}{l}33.5 \pm 2.8^{\star} \\
11.3 \pm 3.0^{\circ}\end{array}$ & $\begin{array}{l}35.2 \pm 5.0^{\star} \\
0.8 \pm 1.7^{\star} \neq\end{array}$ \\
\hline
\end{tabular}

\section{DISCUSSION}

Quantitative measurement of CBF by the Patlak plot method using 99mTc-HMPAO single-photon emission CT is easy to perform, requires no blood sample, and is reliable for clinical evaluation.[2,3,10] Using this method, we observed reduced mean CBF values in patients with both dementia and NPH in previous studies. $[2,3]$ In patients with mild-to-moderate dementia (mean age 67.3 years) a significant reduction in mean $\mathrm{CBF}$ velocity $(36.4 \pm 3.4 \mathrm{ml} / 100 \mathrm{~g} /$ minute) was shown. In patients with severe dementia (mean CBF value $32.6 \pm 2.4 \mathrm{ml} / 100 \mathrm{~g} /$ minute; mean age 73.6 years) a significantly lower ( $\mathrm{p}<$ $0.01)$ mean $\mathrm{CBF}$ value was observed than that in patients with mild-to-moderate dementia. Reduced 
mean CBF was shown preoperatively in patients with NPH, and CBF restoration in patients with clinical improvement suggested that the placement of a shunt relieved CSF diffusion and prevented ischemia in the periventricular white matter.

In the central nervous system, because $\mathrm{CBF}$ is regulated by the regional metabolic demands that depend on neural activity,[13] a lowered CBF can be caused by hypometabolism due to a disruption of neural networks. The fall in CBF may be a secondary phenomenon resulting from a decrease in cerebral metabolism caused by the decrease in volume of brain tissue. In the present study, in patients with or without NPH a significantly reduced mean CBF value was shown, with no difference between the two groups. Therefore, the simple observation of the reduced CBF alone does not identify NPH in patients with dementia and ventriculomegaly. In the four patients with NPH a significant increase in the postoperative mean CBF velocity was not demonstrated, which was different from our previous study,[3] probably because the number of patients was small.

Acetazolamide increases CBF without changing the flow distribution.[14,16,17] Tomographically observed mean CBF increases by $31 \%$ (range 13-46\%) in normal elderly people after an intravenous injection of $1 \mathrm{~g}$ of acetazolamide.[16] However, patients with compromised circulation and resultant reduced $\mathrm{CBF}$ due to reduced perfusion pressure cannot respond to acetazolamide.[19] Although vasomotor responsiveness during inhalation of $100 \%$ oxygen and/or $5 \%$ carbon dioxide in patients with $\mathrm{NPH}$ is impaired before shunt placement, it normalizes after CSF is removed via lumbar puncture or drained via ventriculoperitoneal shunting.[12] In the present study, selection of patients for surgery was unrelated to the results of mean CBF and CVR values. The six patients who improved after placement of a shunt can be considered to have had true NPH. The CVR values measured in these patients were impaired significantly compared with those in the normal controls, and they improved significantly after a shunt was placed. Such impairment of CVR may be due to compression of small-caliber vessels by increased water content and high tissue pressure in the periventricular white matter resulted from increased bulk diffusion of CSF flow.

In contrast, hypometabolic brain tissue unrelated to major vascular diseases may respond to acetazolamide because the cerebral arterioles may be abnormally constricted by a reduction in carbon dioxide production.[11] Thus, in patients with dementia associated with Alzheimer's disease or with senile dementia no impairment of vascular response caused by either oxygen or carbon dioxide inhalation is shown. $[1,8,18]$ In the patient with probable Alzheimer's dementia (Case 12) and in other patients with dementia but no NPH, reduced CBF but preserved CVR was demonstrated, although MR imaging revealed various degrees of white matter lesions. Therefore, the low mean CBF velocity may have been caused by hypometabolism in these patients. The preserved CVR in these patients with dementia and ventriculomegaly suggests that metabolic depression due to progressive brain atrophy or irreversible degenerative alteration unrelated to NPH played an important role in the manifestation of their symptoms.

Cerebrovascular reactivity was extremely impaired in demented patients with NPH but not in those without NPH, although the mean CBF value was reduced in both groups. Reductions in mean CBF and CVR values can be proposed as diagnostic criteria for identifying patients with idiopathic NPH, but further study of a less-selected patient population is required. The findings of the present study suggest that ischemia due to the process of NPH is responsible for the reduction of $\mathrm{CBF}$ and the manifestation of symptoms in patients with idiopathic NPH. 


\section{References}

1. Amano T, Meyer JS, Okabe T, et al: Cerebral vasomotor responses during oxygen inhalation. Results in normal aging and dementia. Arch Neurol 40:277-282, 1983

2. Chang CC, Kuwana N, Noji M, et al: Cerebral blood flow measurement in patients with impaired consciousness: usefulness of 99mTc-HMPAO single-photon emission tomography in clinical practice. Eur J Nucl Med 25:1330-1332, 1998

3. Chang CC, Kuwana N, Noji M, et al: Cerebral blood flow in patients with normal pressure hydrocephalus. Nucl Med Commun 20:167-169, 1999

4. Del Bigio MR, Bruni JE: Changes in periventricular vasculature of rabbit brain following induction of hydrocephalus after shunting. J Neurosurg 69:115-120, 1988

5. Drayer BP, Rosenbaum AE, Higman HB: Cerebrospinal fluid imaging using serial metrizamide CT cisternography. Neuroradiology 13:7-17, 1977

6. Graff-Radford NR, Rezai K, Godersky JC, et al: Regional cerebral blood flow in normal pressure hydrocephalus. J Neurol Neurosurg Psychiatry 50:1589-1596, 1987

7. Kushner M, Younkin D, Weinberger J, et al: Cerebral hemodynamics in the diagnosis of normal pressure hydrocephalus. Neurology 34:96-99, 1984

8. Kuwabara Y, Ichiya Y, Otsuka M, et al: Cerebrovascular responsiveness to hypercapnia in Alzheimer's dementia and vascular dementia of the Binswanger type. Stroke 23:594-598, 1992

9. Mamo HL, Meric PC, Ponsin JC, et al: Cerebral blood flow in normal pressure hydrocephalus. Stroke 18:1074-1080, 1987

10. Matsuda H, Tsuji S, Schuke N, et al: A quantitative approach to technetium-99m hexamethylpropylene amine oxime. Eur J Nucl Med 19:195-200, 1992

11. Matsuda H, Tsuji S, Sumiya H, et al: Acetazolamide effect on vascular response in areas with diaschisis as measured by Tc-99m HMPAO brain SPECT. Clin Nucl Med 17:581-586, 1992

12. Meyer JS, Tachibana H, Hardenberg JP, et al: Normal pressure hydrocephalus. Influences on cerebral hemodynamic and cerebral fluid pressure-chemical autoregulation. Surg Neurol 21:195-203, 1984

13. Sokoloff L: Relationships among local function activity, energy metabolism, and blood flow in the central nervous system. Fed Proc 40:2311-2316, 1981

14. Sullivan HG, Kingsbury TB IV, Morgan ME, et al: The rCBF response to Diamox in normal subjects and cerebral disease patients. J Neurosurg 67:525-534, 1987

15. Tanaka A, Kimura M, Nakayama Y, et al: Cerebral blood flow and autoregulation in normal pressure hydrocephalus. Neurosurgery 40:1161-1167, 1997

16. Vostrup S, Burn B, Lassen NA: Evaluation of the cerebral vasodilatory capacity by the acetazolamide test before EC-IC bypass surgery in patients with occlusion of the internal carotid artery. Stroke 17:1291-1298, 1986 
17. Vostrup S, Henriksen L, Paulson OB: Effect of acetazolamide on cerebral blood flow and cerebral metabolic rate for oxygen. J Clin Invest 67:1634-1639, 1984

18. Yamaguchi F, Meyer JS, Yamamoto M, et al: Noninvasive regional cerebral blood flow measurements in dementia. Arch Neurol 37:410-418, 1980

19. Yonas H, Smith HA, Durham SR, et al: Increased stroke risk predicted by compromised cerebral blood flow reactivity. J Neurosurg 79:483-489, 1993

Manuscript received January 26, 1999.

Accepted in final form September 15, 1999.

Address reprint requests to: Chia-Cheng Chang, M.D., Department of Neurosurgery, Yokohama City University School of Medicine, 3-9 Fukuura, Kanazawa-ku, Yokohama, Japan 236-0004. email nouge@yellow.med.yokohama-cu.ac.jp. 